\title{
Construction of the LISA back-side fibre link interferometer prototype
}

\author{
F Steier ${ }^{1}$, R Fleddermann $^{1}$, J Bogenstahl $^{2}$, C Diekmann $^{1}$, G Heinzel $^{1}$ \\ and K Danzmann ${ }^{1}$ \\ ${ }^{1}$ Max-Planck-Institut für Gravitationsphysik (Albert-Einstein-Institut) and Universität Hannover, \\ Germany \\ ${ }^{2}$ Institute for Gravitational Research, Department of Physics and Astronomy, University of \\ Glasgow, UK \\ E-mail: frank.steier@aei.mpg.de
}

Received 1 April 2009, in final form 28 July 2009

Published 19 August 2009

Online at stacks.iop.org/CQG/26/175016

\begin{abstract}
The Laser Interferometer Space Antenna (LISA) is a joint ESA NASA mission to be launched in 2018. It is an interferometric gravitational wave detector with a measurement band going from $0.1 \mathrm{mHz}$ to $1 \mathrm{~Hz}$. The conceptual interferometer design is unique and includes many challenging aspects that must be analysed in terms of their stability in advance to the mission. One of these new features is the so-called back-side fibre link, which connects the two optical benches on-board each spacecraft. In its optical fibre, two frequency shifted laser beams are counter-propagating. LISA will only reach its design sensitivity, if these two beams inside this fibre experience the same pathlength changes down to a level of approximately $1 \mathrm{pm} / \sqrt{\mathrm{Hz}}$ in the $\mathrm{mHz}$ range. In this paper, we present the construction of a quasi-monolithic interferometer that represents a cutout of the LISA interferometry concerning the back-side fibre link. In order to ensure a high thermal and mechanical stability of the interferometer, the hydroxide-catalysis bonding technique was applied. For the construction of the interferometer, a number of new alignment techniques and solutions were developed that are suitable for LISA prototype experiments.
\end{abstract}

PACS numbers: 99.55.Ym, 04.80.Nn, 07.60.Ly

(Some figures in this article are in colour only in the electronic version)

\section{Introduction}

LISA consists of three satellites forming an equilateral triangle with $5 \times 10^{9} \mathrm{~m}$ armlength. Each spacecraft carries two optical benches with a laser associated with each optical bench. The two lasers must be phase locked with respect to each other with a programmed offset 


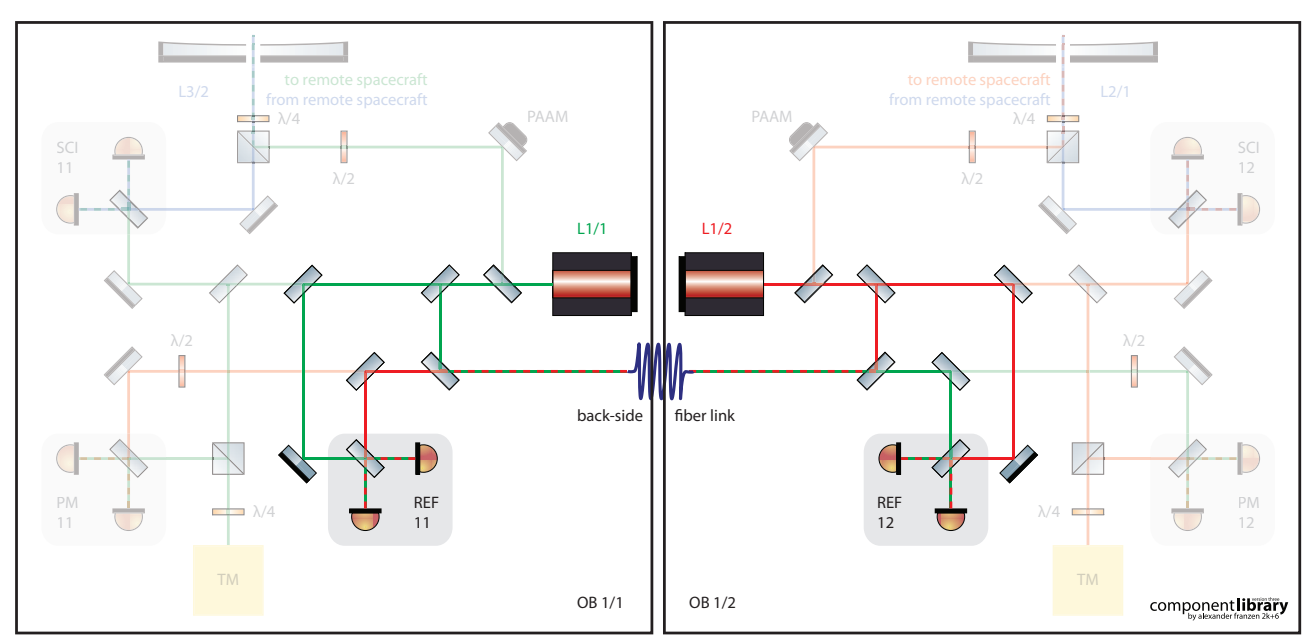

Figure 1. Schematic view of the LISA back-side fibre link interferometer.

frequency. For this purpose, the two optical benches are connected with a fibre in which the two laser beams are counter-propagating. This optical link is called the back-side fibre link. Figure 1 shows a schematic view of the LISA interferometer on-board one of the spacecraft. The part concerning the back-side fibre link is highlighted.

The interferometer baseplates in LISA will be made of ultra-low expansion material and form a quasi-monolithic compound together with the optical components attached to them. A subset of the LISA interferometer concerning the fibre link was constructed as a prototype for the purpose of characterizing the back-side fibre link. This paper concentrates on new aspects in the construction of monolithic interferometers.

For applications associated with LISA, other complex quasi-monolithic interferometers have already been built. The ones known to the authors are the prototype of the LISA Pathfinder optical bench [1] built at the Institute for Gravitational Research at University of Glasgow and its Engineering Model [2] built at the Rutherford Appleton Laboratories with the support of the University of Glasgow and the Albert-Einstein-Institut Hannover. Furthermore, interferometers with similar complexity are presently in planning. These are the Flight Models of the LISA Pathfinder optical bench built at the University of Glasgow [3] and an interferometer also built by the University of Glasgow which will be used for verification of the performance of polarizing optics for the LISA interferometry [4]. Furthermore, another interferometer is in planning at the Albert-Einstein-Institut Hannover that also characterizes polarizing components, but focuses more on a different space mission that will map the gravity field of the Earth [5].

\section{Design of the back-side fibre link breadboard}

The layout of the back-side fibre link interferometer is shown in figure 2. It includes three main interferometers, two auxiliary interferometers and is aligned for two different beam path at the same time. The interferometers are of type Mach-Zehnder. The modulation and readout for the interferometer is realized in the same way as for LISA Pathfinder [6]. The two beams injected (labelled as BEAM 1 and BEAM 2 in figure 2) interfere in the reference interferometer which is read out by the photodiodes PDR1 and PDR2. The reference interferometer measures 


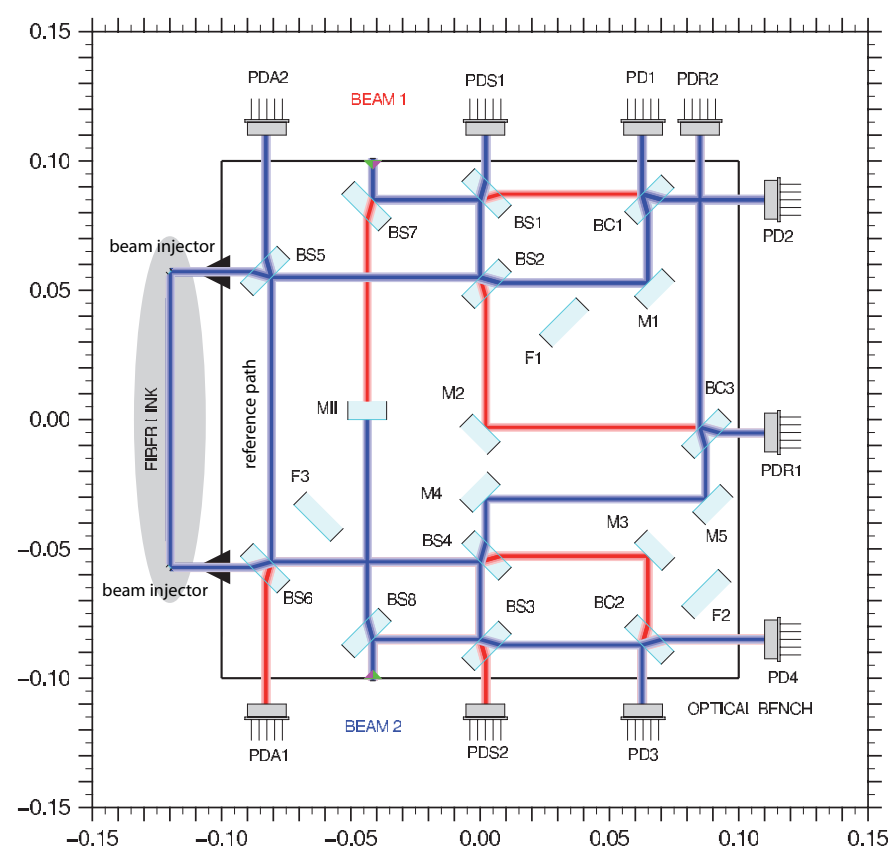

Figure 2. Interferometer layout of the back-side fibre link interferometer breadboard.

the pathlength fluctuations of the unstable part of the interferometer, where the two injected beams are prepared. The unstable paths are common for all interferometers. The beams are split only on the ultra-stable optical bench so that the reference interferometer can be used to cancel the common mode fluctuations by subtracting them from all other interferometer outputs.

The rest of the set-up is symmetric. The upper half corresponds to one of the LISA optical benches and the lower half to the second LISA optical bench on-board each of the spacecraft. In both halves, there is one main measurement interferometer. On the upper half, beam 1 is routed directly to the beamsplitter $\mathrm{BC} 1$, while beam 2 is routed through the fibre link (via BS8, BS3 BS4, BS6, fibre, BS5, BS2 and M1) before arriving at this beamsplitter. Here, the pathlength fluctuations of the fibre are measured in one direction. On the lower half, beam 2 is routed directly to BC2 and beam 1 passes through the fibre. Here, the pathlength fluctuations introduced by the fibre are measured in the opposite direction. By combining these measurements, the differential pathlength changes of the two counter-propagating beams can be measured [7].

An additional useful feature of the interferometer is a path that can be used instead of the fibre. It is implemented by the reflected beams of BS5 and BS6 which shortcut the fibre. When using this path, the instrument can be characterized in terms of its sensitivity without the fibre under test.

One important noise source in this interferometer turned out to be introduced by backreflection at the fibre injectors of the fibre link. These reflections introduce spurious beat signals in the measurement interferometers that reduce the interferometer sensitivity [7, 8]. Using the photodiodes PDS1 and PDS2 these spurious beat signals can be measured separately with the same amplitude and phase as they appear in the measurement interferometers. Therefore, these auxiliary interferometers can be used to investigate the influence of this effect on the main measurement. 


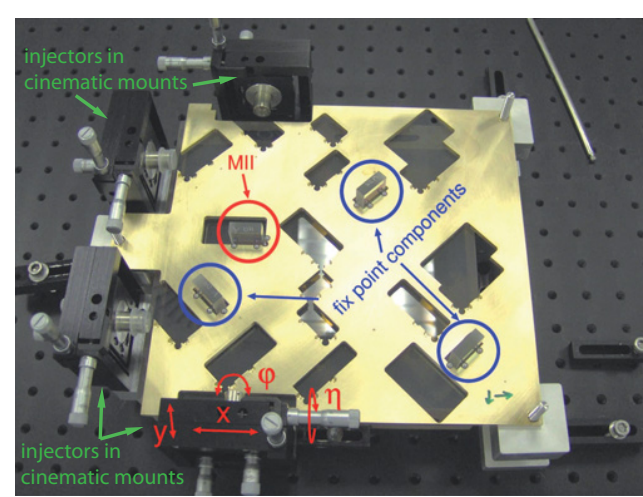

(a) Beam injectors and first components.

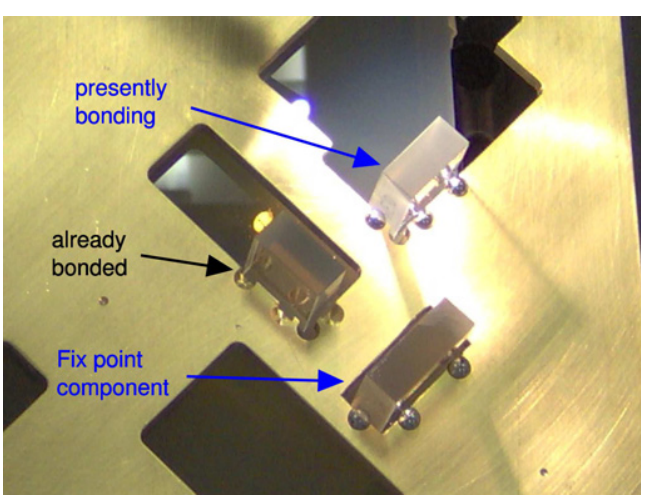

(b) Pockets of the template.

Figure 3. The template used for the adjustment of components that are less critical in terms of alignment. (a) Beam injectors and first components. (b) Pockets of the template.

\section{Alignment methods}

Some alignment methods applied for the construction of the back-side fibre link interferometer are partly copied from other monolithic interferometers mentioned in section 1 but, additionally, new techniques and features were applied. They are discussed in more detail in the following step-by-step description of the construction procedure.

\subsection{Input beam alignment}

The construction of the interferometer started with the attachment of the input beam injectors shown in figure $3(a)$. The injectors were inserted into compact kinematic mounts with an overall width of less than $10 \mathrm{~cm}$ and with $4^{\circ}$ of freedom. The alignment of the input beams is thus possible in 2 angular and 2 lateral degrees of freedom. In particular, it is necessary to optimize the alignment of the input beams in height, $y$, and horizontal angle, $\varphi$, since wrong input beam alignment in these degrees of freedom cannot be compensated by the alignment of the bonded components. The mounts are directly attached to the side of the baseplate using an epoxy resin (Electrotube, ER2188) with low thermal expansion.

The mounts of the input beam injectors turned out to be very handy. In contrast to other bonded interferometers, these injectors can be aligned in all important degrees of freedom and are directly attached to the baseplate. In earlier designs, the beam injectors were either placed beside the baseplate on a common breadboard or directly attached to the baseplate but due to the requirement of providing ultra-stable fibre injectors for the optical bench without the ability of realignment.

Since the bonded components do not allow alignment of the vertical beam angle, the bonding components have a maximum deviation from perpendicularity of 1 arcsecond between the optical surface and the bottom surface. Therefore, it must be ensured that the input beams are aligned to be parallel with respect to the baseplate. In the presented interferometer, this is realized by reflecting beam 2 (the fibre injector shown at the bottom in figure 3(a)) back into itself using the mirror $\mathrm{M}_{\|}$. By measuring and maximizing the light power that is coupled back into the fibre injector, the parallelism between the input beam and the baseplate was optimized within the accuracy of the perpendicularity of the mirror. This new method of input beam 

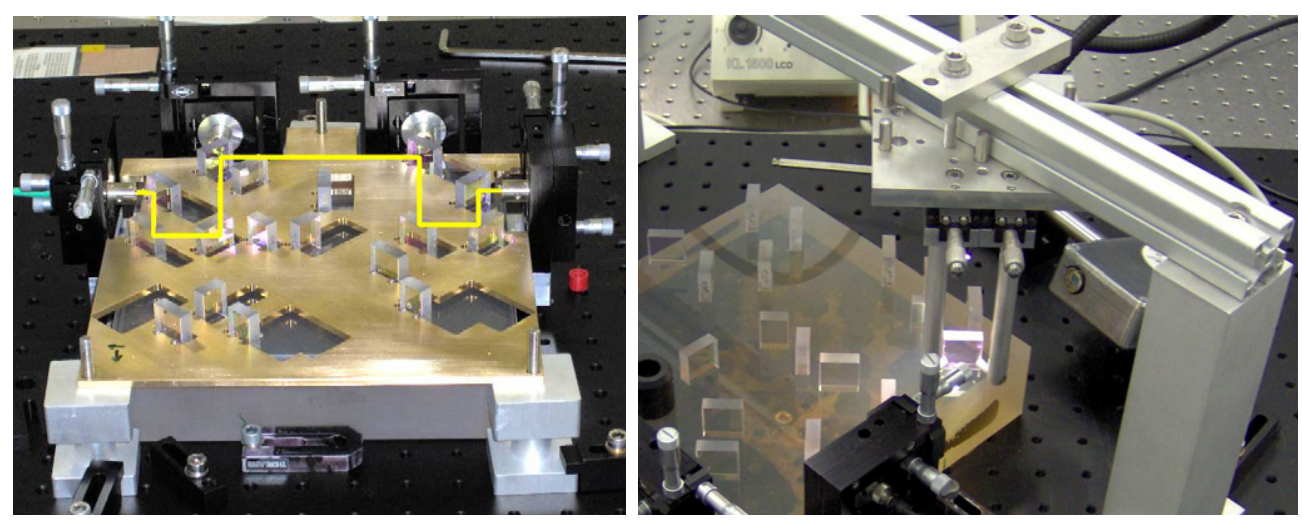

Figure 4. Critical alignment steps: left: beam injectors and null measurement path, right: recombination beamsplitter alignment.

alignment was demonstrated to be successful and could be used for further construction of optical benches for LISA-related experiments.

\subsection{Component alignment}

The components of the interferometer can be grouped into moderately critical and very critical in terms of their alignment requirements. All mirrors and beamsplitters that only split but not recombine the laser beams are moderately critical. These components were aligned using a mechanical template.

A photograph of the template is shown in figure 3 with a close-up view in figure $3(b)$ showing the pockets that are foreseen for each of the optical components. The template is made of brass with a machining accuracy of approximately $100 \mu \mathrm{m}$. At the edge of these pockets, there are three cutouts that can hold spheres to define an exact 3-point reference.

The same template was used for several bonding steps and had to be removed several times. In order to place the template always at the same position in each bonding step, three bonded components were foreseen (labelled as fix point components in figure $3(b)$ ) which are not used optically, but only as mechanical references for the template positioning. They were bonded as the first set of optical components (figure 3(a)). These fix point components have deliberately small pockets. Thus, it was impossible to move the template in a way that it breaks off components that had already been bonded on the baseplate.

\subsection{Fibre injector and null-measurement path alignment}

One novel step in the bonding process was the alignment of the fibre injectors of the fibre link, and the null-measurement path that can substitute the fibre path. The left-hand side of figure 4 shows this path highlighted in a photograph of the interferometer. The beam coming from the injector on the left-hand side is the one that had already been aligned in the initial step. Due to the interferometer topology, there is a path to the second beam injector both through the fibre and via the null-measurement path. By adjusting the opposite injector with respect to the beam from the first injector travelling via the null-measurement path, it was made to be parallel to the baseplate and on the same height as the first one. Thereby both input beams were aligned with respect to each other.

On the left-hand side of figure 4, the two fibre injectors of the back-side fibre link are shown in the back. By aligning them with respect to the beams coming from the optical 


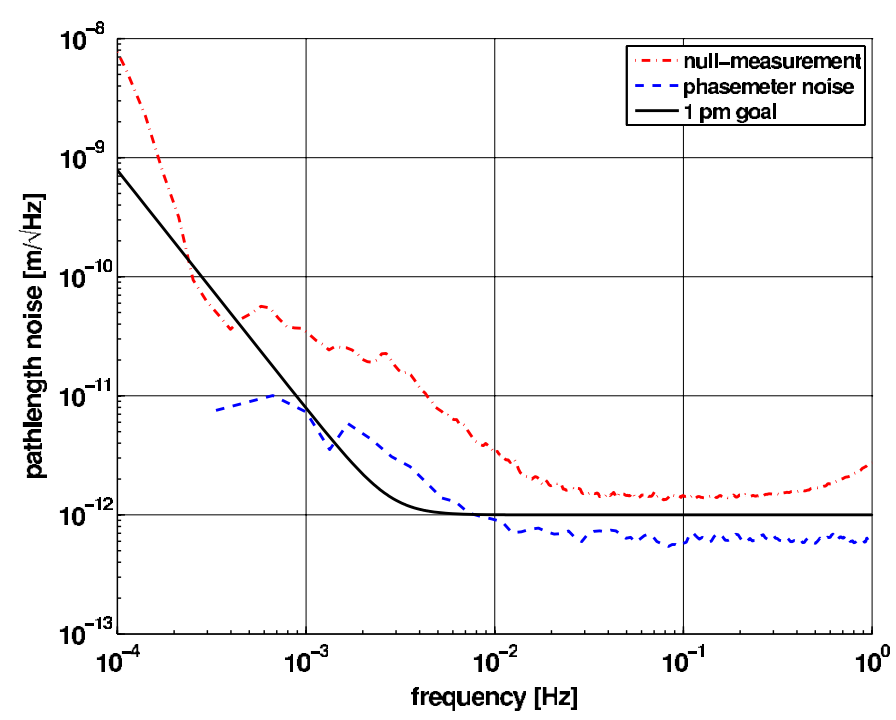

Figure 5. Interferometer sensitivity achieved using the null-measurement path of the back-side fibre link interferometer.

bench, their output beams and the beams that travel through the null-measurement path are automatically aligned to be on the same beam axis. Thus the recombination beamsplitter alignment, described in the next step, is optimized for both the beam travelling through the fibre and the beam travelling through the null-measurement path. Therefore, either of the two different paths can be used without realignment of the interferometer.

\subsection{Recombination beamsplitter alignment}

The back-side fibre link interferometer prototype includes three main interferometers. Their recombination beamsplitters $(\mathrm{BC} 1, \mathrm{BC} 2$ and $\mathrm{BC} 3)$ must be aligned while the interference signals are monitored and the contrast is optimized. The final alignment of these beamsplitters must be done before the bond hardens which was here a time interval of less than 1 min. Otherwise the components stick to the baseplate in a non-optimal position and it is not possible to remove them without damaging the surface of the baseplate. Thus, an alignment tool was constructed to facilitate this critical adjustment. The tool is shown on the right-hand side of figure 4. It consists of a bridge with a movable console that was placed above the baseplate. The console has two fingers with micrometer positioners that allow the lateral and angular component alignment. Similar tools have also been used for the construction of other bonded interferometers $[1,2]$.

In order to optimally adjust each interferometer, the interference signal was monitored during the recombination beamsplitter alignment and the contrast was optimized. The contrasts value achieved in the individual interferometers were approximately $80 \%$ of the theoretical maximum. This is comparable to the contrast reached in the LISA Pathfinder Engineering Model.

\section{Interferometer stability}

The interferometer stability achieved so far is shown in figure 5. The uppermost curve corresponds to the differential pathlength changes when the null-measurement path is used. 
For comparison, the readout noise is shown as the dashed line. The phasemeter noise is measured by combination of two different readout channels with the same input signal. Above about $20 \mathrm{mHz}$ the stability is below $2 \mathrm{pm} / \sqrt{\mathrm{Hz}}$. This is only slightly worse than, e.g., the performance of the LISA Pathfinder Engineering Model optical bench [9] which shows the best performance in the LISA measurement band of bonded interferometers up to now. The excess noise is still not completely understood and investigations are ongoing. The set-up was first optimized for the null-measurement in order to have a testbed with sufficient sensitivity. Measurements with the fibre are not yet comparable to that shown in figure 5.

The sensitivity shown is an upper limit for the stability of an interferometer that can be achieved using the techniques described above. Further optimizations of the readout are investigated which might lead to a reduction of the observed excess noise.

\section{Conclusion}

New alignment techniques in the application of hydroxide-catalysis bonding were used for the construction of the back-side fibre link interferometer prototype. These techniques result particularly in a considerably simple input beam alignment. Furthermore, specific diagnostic features like the null-measurement path were included that offer the ability of comprehensive instrument characterization.

The baseplate includes three main interferometers that, in combination, measure the fibre characteristics, and two interferometers that measure the null-measurement path instead of the fibre. This null-measurement path and the path through the fibre are exchangeable while the interferometers remain always optimally aligned for both. In addition, it has two auxiliary interferometers to characterize the back-reflections at the fibre injectors of the back-side fibre link.

The excess noise of the sensitivity achieved up to now is not yet completely understood and investigations are ongoing. The sensitivity achieved of less than $2 \mathrm{pm} / \sqrt{\mathrm{Hz}}$ between $20 \mathrm{mHz}$ and $1 \mathrm{~Hz}$ gives an upper limit for the bonding techniques applied. Down to this level, the techniques are sufficient for LISA breadboard experiments.

\section{Acknowledgments}

The application of the hydroxide-catalysis bonding technique was intensively supported by the Institute for Gravitational Research of the University of Glasgow. We appreciate their support. We also gratefully acknowledge support by Deutsches Zentrum für Luft- und Raumfahrt (DLR) (references 50 OQ 0501 and 50 OQ 0601).

\section{References}

[1] Elliffe E J et al 2005 Class. Quantum Grav. 22 S257

[2] Middleton K F et al 2006 Opt. Eng., Bellingham 45125601

[3] Killow C F et al 2006 AIP Conf. Proc. 873 S297

[4] Fitzsimons E et al 2009 AIP Conf. Proc. 154012034

[5] Dehne M et al 2009 AIP Conf. Proc. 154012023

[6] Heinzel G et al 2003 Class. Quantum Grav. 20 S153

[7] Steier F 2008 Interferometry techniques for spaceborne gravitational wave detectors PhD Thesis Universität Hannover, Germany

[8] Fleddermann R et al 2009 AIP Conf. Proc. 154012022

[9] Heinzel G et al 2005 Class. Quantum Grav. 22 S149 